\title{
Study of Rare Cases of Congenital Myasthenic Syndrome in Bangladesh
}

\author{
BANU $A^{1}$, RAHMAN MM ${ }^{2}$, ANWAR MM $^{3}$, AHMED R ${ }^{4}$, AZAD MAK ${ }^{5}$, KABIR J $^{6}$, AHMED A $^{7}$, \\ SARKAR $D^{8}$, JAHAN SMS ${ }^{9}$, OHAB MA $^{10}$, ALAM MS $^{11}$, HAQUE MA $^{12}$, SHAHEDUZZAMAN AKM ${ }^{13}$, \\ RAHMAN MM ${ }^{14}$, MONDOL SI ${ }^{15}$, SARKAR MK ${ }^{16}$, MIAH MH ${ }^{17}$, MONDOL R $^{18}$, RABBANI MG ${ }^{19}$, \\ PONDIT PK ${ }^{20}$, SARKAR NC ${ }^{21}$, SARKAR MRA ${ }^{22}$, AZAD AK $^{23}$, ADILUZZAMAN MAFA $^{24}$, \\ KARMOKAR P ${ }^{25}$, SUTRADHAR P ${ }^{26}$, MAMOON MA ${ }^{27}$, MONDOL $F^{28}$, SHAFIUJJAMAN ASM $^{29}$,
}

\begin{abstract}
:
Congenital myasthenic syndromes (CMS) comprise a heterogeneous group of rare inherited diseases in which the neuromuscular transmission in the motor plate is compromised by one or more genetic pathophysiological specific mechanisms are characterized by fatigable weakness of skeletal muscle (e.g., ocular, bulbar, limb muscles) with onset at or shortly after birth or in early childhood; rarely, symptoms may not manifest until later in childhood. The diagnosis of CMS is based on clinical findings, a decremental EMG response of the compound muscle action potential (CMAP) on low-frequency (2$3 \mathrm{~Hz}$ ) stimulation, absence of anti-acetylcholine receptor (AChR) and anti-MuSK antibodies in the serum, a positive response to acetylcholinesterase (AchE) inhibitors and lack of improvement of clinical symptoms with immunosuppressive therapy. Pathogenic variants in one of multiple genes encoding proteins expressed at the neuromuscular junction are currently known to be associated with subtypes of CMS. The most commonly associated genes include: CHAT, CHRNE, COLQ, DOK7, GFPT1 and RAPSN. We studied on a sibling presented with progressive fatigability and fluctuating ptosis with frequent exacerbations of muscle weakness during infections since infancy. On both cases CT scan of chest were negative for thymoma, antibodies
\end{abstract}

1. Dr. Akter Banu, Assistant Professor, Department Of Pediatrics, Rangpur Medical College, Rangpur.

2. Dr. Md. Mahfuzer Rahman, Professor, Department Of Medicine, Rangpur Medical College, Rangpur.

3. Dr. Md Mahfuj UI Anwar, Assistant Professor, Department Of Medicine, Rangpur Medical College, Rangpur.

4. Dr. Raihan Ahmed, Indoor Medical Officer, Department of Medicine, Rangpur Medical College Hospital, Rangpur.

5. Dr. Md. Abul Kalam Azad, Assistant Professor, Department of Medicine, Rangpur Medical College, Rangpur.

6. Dr. Jahangir Kabir, Assistant Professor, Department of Medicine, Rangpur Medical College, Rangpur.

7. Dr. Anis Ahmed, Assistant Professor, Department of Neurology, BSMMU, Dhaka.

8. Dr. Devendranath Sarkar, Professor, Department of Medicine, Rangpur Medical College, Rangpur.

9. Dr. Shah Md. Sarwer Jahan, Professor, Department of Medicine, Rangpur Medical College, Rangpur.

10. Dr. MA Ohab, Associate Professor, Department of Medicine, Rangpur Medical College, Rangpur.

11. Dr. Shafiul Alam, Associate Professor, Department of Medicine, Rangpur Medical College, Rangpur.

12. Dr. Md. Ashraful Haque, Associate Professor, Department of Medicine, Rangpur Medical College, Rangpur.

13. Dr. AKM Shaheduzzaman, Associate Professor, Department of Medicine, Rangpur Medical College, Rangpur.

14. Dr. Md. Mokhlesur Rahman, Associate Professor, Department of Medicine, Rangpur Medical College, Rangpur.

15. Dr Shariful Islam Mondol, Associate Professor, Department of Medicine, Rangpur Medical College, Rangpur.

16. Dr. Md. Kamruzzaman Sarkar, Associate Professor, Department of Medicine, Rangpur Medical College, Rangpur.

17. Dr. Md. Helal Miah, Assistant Professor, Department of Medicine, Rangpur Medical College, Rangpur.

18. Dr. Rahenur Mondol, Assistant Professor, Department of Medicine, Rangpur Medical College, Rangpur.

19. Dr. Md. Golam Rabbani, Assistant Professor, Department of Medicine, Rangpur Medical College, Rangpur.

20. Dr. Prosanto Kumar Pondit, Assistant Professor, Department of Neurology, Rangpur Medical College, Rangpur.

21. Dr. Narayan Chandro Sarkar, Assistant Professor, Department of Medicine, Rangpur Medical College, Rangpur.

22. Dr. Md. Ruhul Amin Sarkar, Assistant Professor, Department of Medicine, Rangpur Medical College, Rangpur.

23. Dr. Abul Kalam Azad Assistant Professor, Department of Medicine, Rangpur Medical College, Rangpur.

24. Dr. Md. Al Fatah Al Adiluzzaman, Assistant Professor, Department of Medicine, Rangpur Medical College, Rangpur.

25. Dr. Priobrata Karmakar, Assistant Professor, Department of Medicine, Rangpur Medical College, Rangpur.

26. Dr. Probal Sutradhar, Specialist (Medicine), Haragach 31 Beded Hospital,Rangpur.

27. Dr. Md. Abdullah Al Mamoon, Indoor Medical Officer, Department of Medicine, Rangpur Medical College Hospital, Rangpur.

28. Dr. Firoz Mondol, Registrar, Resident, Internal Medicine, Rangpur Medical College, Rangpur

29. Dr. ASM Shafiujjaman, Registrar, Medicine, Rangpur Medical College, Rangpur. 
against the acetylcholine receptor (AChR) and the muscle specific kinase (MuSK) were negative and decremental response on electrophysiological study of Repetitive nerve stimulation (RNS) and EMG were consistent with disease of neuromuscular junction (post synaptic) and they were only on pyridostigmine for long time with marked improvement of symptoms and signs. Considering all scenario both of our cases mostly fits with the autosomal recessive, post synaptic CMS associated with Rapsyn deficiency.

Objective : As in Bangladesh, there is inadequate data on the epidemiological profile of CMS, our aim is to describe these cases for their rarity and the difficulty encountered in diagnosis as they are easily confused with Juvenile Myasthenia Gravis (JMG) and familial myopathies. As both the cases are very rare, it should be an original article.

Key word: Progressive fatigability, Repetitive Nerve stimulation, Auto-antibodies, Congenital Myasthenic Syndrome.

Introduction:

Congenital myasthenic syndromes (CMS) are a heterogeneous group of early-onset genetic neuromuscular transmission disorders due to mutations in proteins involved in the organisation, maintenance, function, or modification of the motor endplate. CMS are clinically characterised by abnormal fatigability, or transient or permanent weakness of extra-ocular, facial, bulbar, truncal, respiratory or limb muscles ${ }^{1}$. Congenital myasthenic syndromes can be classified according to the pattern of inheritance, based on the altered protein involved in the motor plate, or by taking into account the site at the neuromuscular junction (pre-synaptic, synaptic, or postsynaptic) involved with the dysfunction².

The age of onset, severity of presenting symptoms, and distribution of muscle weakness can vary from one patient to another. Congenital myasthenic syndromes (CMS) are characterized by fatigable weakness of skeletal muscle (e.g., ocular, bulbar, limb muscles) with onset at or shortly after birth or in early childhood; rarely, symptoms may not manifest until later in childhood. Severity and course of disease are highly variable, ranging from minor symptoms to progressive disabling weakness ${ }^{3}$. In some subtypes of CMS, myasthenic symptoms may be mild, but sudden severe exacerbations of weakness or even sudden episodes of respiratory insufficiency may be precipitated by fever, infections, or excitement. Cardiac and smooth muscle are usually not involved. Coordination, sensation, and tendon reflexes are normal; cognitive skills are usually normal. A variety of additional symptoms affecting other organ systems can be present in specific subtypes. Some myasthenic symptoms are present at birth. Respiratory insufficiency with sudden apnea and cyanosis are common findings in neonates. Neonates with CMS can have multiple joint contractures (often described as arthrogryposis multiplex congenita [AMC]) resulting from a lack of fetal movement in utero. Other major findings in the neonatal period may include feeding difficulties, poor suck and cry, choking spells, eyelid ptosis, and facial, bulbar, and generalized weakness. Stridor in infancy may be an important clue to CMS. Individuals with onset later in childhood show abnormal muscle fatigability, with difficulty in running or climbing stairs. Motor milestones may be delayed. Affected individuals present with fluctuating eyelid ptosis and fixed or fluctuating extraocular muscle weakness. Ptosis may involve one or both eyelids. In addition, facial and bulbar weakness with nasal speech and difficulties in coughing and swallowing may be present. Spinal deformity or muscle atrophy may occur ${ }^{4}$. Most CMS are transmitted by autosomal recessive inheritance ${ }^{3}$.

A generic diagnosis of a CMS can be made on clinical grounds from a history of fatigable weakness involving ocular muscles, bulbar muscles (muscles of the face, and muscles used for speaking and swallowing), and limb muscles since infancy or early childhood, a history of similarly affected siblings, and a variety of tests. Such tests include a decremental response on Repetitive Nerve stimulation (RNS) and Electromyography (EMG), and negative tests for antibodies against the acetylcholine receptor 
(AChR) and the muscle specific kinase (MuSK). Genetic diagnosis of the CMS is important because therapy that benefits one type CMS may worsen another type ${ }^{3}$.

The prevalence of CMS is very difficult to estimate due to the clinical variability of cases and the fact that many cases have no specific etiologic diagnosis or are undiagnosed. Since they are rare medical conditions in which definite diagnosis rests on clinical, electromyography and specific genetic testing, few data are available. Furthermore, there are few series of patients where this complete diagnostic profile has been established, and most of the current knowledge has been obtained by reports of isolated case reports ${ }^{5}$. According to a recent review, the prevalence of CMS is estimated as $1 / 10$ that of myasthenia gravis, which is $25-$ $125 / 1000000^{6}$. A study in the UK estimated that the prevalence of CMS with a defined genetic diagnosis is approximately 9.2 cases per million children under 18 years old in the population ${ }^{7}$. An important epidemiological profile on CMS was obtained in a study performed at the Mayo Clinic. Most cases occurred as a consequence of postsynaptic defects $(68 \%)$, and basal lamina defects $(13.7 \%)$, development and maintenance of the end plate defects (12.5\%), pure presynaptic defects $(5.9-8 \%)$ and congenital myopathies with secondary neuromuscular junction transmission defects $(0.3 \%)$ also represent other rare congenital myasthenic syndromes. Thus, postsynaptic forms represent up to $75-80 \%$ of all CMS cases ${ }^{5,8,9}$. In Western or central Europe the RAPSN variant c. $264 \mathrm{C}>\mathrm{A}$ and the DOK7 variant C. 1124 1172 dupTGCC are highly prevalent ${ }^{10}$. In a study of $34 \mathrm{CMS}$ families from Israel the genes most frequently mutated were RAPSN $(n=13)$, COLQ $(n=11)$, and CHRNE $(n=7)^{11}$. All other mutated proteins may contribute with less than $1 \%$ of the CMS cases to the general group of CMS. About $75 \%$ of the CMS cases are due to mutations in genes that encode different subunits of the acetylcholine receptor (CHRNA1, CHRNB1, CHRND, CHRNE) or proteins important to maintain the structure or function of the NMJ, such as MUSK, RAPSN or DOK $7^{12,13}$. The most common causative genes are CHAT, COLQ, RAPSN, CHRNE, DOK7, and GFPT1 1 .
The clinical picture of congenital myasthenic syndromes (CMS) is similar to that of myasthenia gravis (MG), in which individuals have a history of fatigable weakness involving ocular, bulbar, and limb muscles; however, the myasthenic symptoms of CMS usually start at or shortly after birth rather than in adulthood, as is usual for MG. Because seronegative autoimmune MG has been reported on occasion in children younger than age two years, MG may be difficult to differentiate from CMS, especially in later childhood or adulthood. Furthermore, immunosuppressive therapy does not improve clinical symptoms in CMS, whereas it does in MG. Other disorders partially resembling CMS at childhood to consider are Spinal muscular atrophy, Congenital muscular dystrophies, Congenital myopathies including X-linked myotubular myopathy nemaline myopathy and multiminicore myopathy, Infantile myotonic dystrophy type 1,Mitochondrial myopathies, Brain stem anomalies, Mobius syndrome, Infantile botulism ${ }^{4}$.

To establish the extent of disease and needs in an individual diagnosed with congenital myasthenic syndromes (CMS), the following evaluations are recommended: Assessment of strength and motor function; in children, assessment of motor, speech, and cognitive development. Assessment of respiratory function with baseline pulmonary function tests including forced vital capacity in sitting and supine positions and blood gas exchange. Polysomnography to identify individuals with nocturnal hypoventilation.Assessment of contractures and joint deformities by physiatrists and orthopedists; radiologic examinations if spinal deformity is observed. Speech therapy evaluation if dysarthria and/or hypernasal speech is present. For early-onset forms, assessment of feeding abilities (sucking, swallowing, gastroesophageal reflux)and growth parameters to determine the need for feeding interventions such as gavage feeding or gastrostomy insertion ${ }^{4}$.

There are no recent published consensus guidelines for the management of CMS treatment. The choice of medical treatment varies with the 
CMS subtype. Therefore, it seems reasonable to consider a first-linegenetic test to evaluate the genetic subtype. Most individuals with $\mathrm{CMS}$ benefit from Acetylcholineesterase (AChE) inhibitors (pyridostigmine) and/or the potassium channel blocker 3,4-diaminopyridine (3,4-DAP); however, caution must be used in giving 3,4-DAP to young children and individuals with fast-channel CMS (FCCMS). Individuals with COLQ and DOK7 pathogenic variants usually do not respond to longterm treatment with AChE inhibitors. Some individuals with slow-channel CMS (SCCMS) are treated with quinidine, which has some major side effects and may be detrimental in individuals with AChR deficiency. Fluoxetine is reported to be beneficial for SCCMS. Ephedrine and albuterol have been beneficial in several individuals, especially as a therapeutic option for those with $D O K 7$ or $C O L Q$ pathogenic variants. In addition to medical therapy, a multidisciplinary approach to the clinical management of the affected individual greatly improves quality of life and can influence survival. Management should be tailored to each individual, their specific CMS subtype, and rate of progression. Depending on the individual clinical situation the clinical management may include the Physical and occupational therapy, speech therapy, orthotics or a wheelchair, a percutaneous gastric tube, ventilator support and genetic counseling 4

\section{Case presentation:}

A 14 years old boy (case 1) from Bangladesh, born of consanguineous parents presented with progressive fatigability and fluctuating ptosis, more marked after exercise. The illness started at the age of 1.5 years. Initially his parents noticed delayed motor developmental milestones such as sitting, walking, feeding difficulties and then ptosis added. He suffered frequent episodes of respiratory tract infections since infancy with exacerbation of muscle weakness during infections. At the age of 9 years, he was diagnosed as a case of Congenital Myasthenic Syndrome (CMS) on the basis of history and clinical examinations and prescribed with pyridostigmine and noticed marked improvement of symptoms with reappearance of symptoms on stopping of medication but he did not get any immunosuppressive therapy including corticosteroid, plasma exchange or thymectomy. Clinical examination of the boy revealed normal intellectual function, nasal voice, elongated face, high arched palate, bilateral partial ptosis, generalized muscle wasting, hypotonia, intact superficial and deep reflexes, waddling gait, kyphosis, hyperlordosis, laxed skin, no external ophthalmoplegia.

His younger sister (case 2), who was 5 years old also presented with progressive fatigability more marked after exercise and delayed motor developmental milestones such as sitting, walking, feeding difficulties and then ptosis since the age of 3 years. None of their other family members suffered from similar illness. She had also been on pyridostigmine for 2 year with marked improvement of symptoms and signs. Clinical examination of the girl revealed normal intellectual function, nasal voice, high arched palate, bilateral partial ptosis, generalized muscle wasting, hypotonia, intact reflexes, waddling gait, laxed skin, no external ophthalmoplegia.

Bed sided tests (Cogan's lid twitch sign, curtain sign, ice on eye test, tensilon test) were positive for myasthenia in both patients.

On investigations similar findings were recorded on both cases. Routine blood examinations were normal. Liver function test, renal function test, creatine kinase (CK) were normal. ECG, Echocardiogram were within normal limit. CT scan of chest was negative for thymoma. Antibodies against the acetylcholine receptor (AChR) and the muscle specific kinase (MuSK) were negative and decremental response on electrophysiological study of Repetitive nerve stimulation (RNS) and EMG were consistent with disease of neuromuscular junction (post synaptic).

\section{Discussion:}

At present there are no well-defined diagnostic criteria for CMS. Congenital myasthenic syndromes should be suspected in cases of: (i) early-onset fatigable muscle weakness mainly involving ocular, bulbar and proximal limb musculature (generally varying from birth to late childhood); (ii) a positive family history of a specific disorder or sometimes only the history of a 
hypotonic infant; (iii) clinical and neurophysiological myasthenic findings with a negative antibody testing profile; (iv) electromyography (EMG) studies showing decremental responses of $10 \%$ or more in the amplitude or in the quarter of the area from the first evoked compound motor action potential (CMAP), or single-fiber EMG studies compatible with a neuromuscular junction dysfunction; and (v) the presence of a specific clinical syndromic phenotype (i.e. Escobar syndrome, Pierson syndrome $)^{9,14,15}$. Both of our cases fulfilled most of the above mentioned criteria.

Although cases of myasthenia gravis during infancy and childhood such as Juvenile Myasthenia Gravis (JMG) have been described in the literature since $1960,{ }^{16-18}$ the distinction between acquired autoimmune form and congenital forms has been increasingly recognized and emphasized ${ }^{19-28}$. This increasing awareness regarding congenital forms of myasthenia gravis was originally described in a previous study described the nosology of congenital myasthenic syndrome ${ }^{29}$. The current thrust of research is naturally directed towards elucidation of molecular basis of such disorders ${ }^{30-35}$. Two major features distinguish CMS from acquired autoimmune myasthenia gravis (MG) are a positive family history and absence of AChR antibodies. In this study both patients are siblings having absence of Anti-AchR and Anti-MuSK antibodies. While a positive family history is consistent with the diagnosis of CMS, a negative family history does not exclude autosomal recessive inheritance, an incompletely penetrant autosomal dominant gene in one parent, or a new mutation ${ }^{36}$. Furthermore, while most cases of acquired autoimmune childhood MG are sporadic, familial aggregates have been observed which may be due to inheritance of HLA haplotypes that predispose to sensitisation of acetylcholine receptor (AChR) ${ }^{36}$. On the other hand, while a positive AChR antibody test excludes the diagnosis of CMS, but a negative test in a sporadic case does not necessarily imply a diagnosis of CMS because a high proportion of juvenile patients with autoimmune MG are also seronegative ${ }^{37}$. It is important to note here that correct differentiation of CMS from autoimmune MG is also important therapeutically, as CMS patients do not respond to immunosuppressive therapy or plasma exchange and thymectomy ${ }^{38}$. Treatment of JMG commonly includes anticholinesterases, corticosteroids and newer immune modulating agents, plasma exchange and intravenous immunoglobulin (IVIG) and thymectomy ${ }^{39}$. Acetylcholinesterase inhibitor is the first line therapy may be sufficient in ocular JMG or mild generalised JMG ${ }^{40}$. Frequently some form of immunosuppression or immunomodulation is required to improve symptoms of JMG. Corticosteroids are often effective and sometimes are the mainstay of therapy ${ }^{41}$. A systematic review of the literature concluded that thymectomy increases the probability of remission or improvement of symptoms in AChR seropositive, nonthymomatous, autoimmune $\mathrm{MG}^{42}$.In our study both of the patients were on pyridostigmine for long time noticing marked improvement of symptoms with reappearance of symptoms on stoppage of drug. And neither of the patients needed any immunosuppressive therapy, plasma exchange or thymectomy. Such scenario goes in favor of CMS rather than Autoimmune MG.

A temporary form of MG affects $10 \%$ to $20 \%$ of newborns whose mothers have immune-mediated MG known as Transient Neonatal Myasthenia Gravis (TNMG) ${ }^{43}$. Our cases did not have any history of maternal MG.

Most CMS are transmitted by autosomal recessive inheritance ${ }^{3}$. CMS due to mutations within the RAPSN gene is an autosomal recessive (AR) disorder ${ }^{44}$. The consanguinity of parents, unaffected parents of our cases are suggestive of AR inheritance.

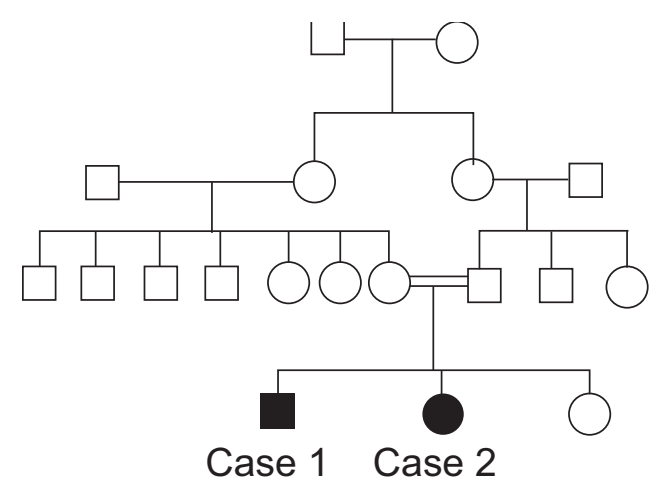


On EMG or RNS studies, CMS show decremental response of $10 \%$ or more in the amplitude or in the quarter of the area from the first evoked compound motor action potential (CMAP), or single-fiber EMG studies compatible with a neuromuscular junction dysfunction $8,14,15$. Postsynaptic forms represent up to $75-80 \%$ of all CMS cases ${ }^{5,8,9}$. Both of our patients' decremental response on electrophysiological study of RNS and EMG were consistent with disease of neuromuscular junction (post synaptic).

Postsynaptic CMS are subdivided into Primary AChR deficiency, Kinetic abnormalities of the AChR (such as Fast Channel CMS \& slow channel CMS) and defects within the AChR clustering pathway (specially CMS associated with deficiency of Rapsyn and Plectin) ${ }^{1,45}$. Slow channel CMS is an autosomal disorder and deterioarates with AChR inhibitors ${ }^{38}$. CMS associated with deficiency of Plectin is refractory to pyridostigmine ${ }^{2}$. On the other hand, primary AChR deficiency, fast channel syndrome, Rapsyn deficiency are well responsive to pyridostigmine. Fast channel CMS (FCCMS) usually present in early childhood with infantile phenotype 46. FCCMS arises from autosomal recessive mutations in different domains of the acetylcholine receptor subunits and clinically mimicks a typical autoimmune acquired myasthenia gravis starting in the first decade of life with good clinical response to treatment with pyridostigmine and amiframpidine ${ }^{2}$. Primary deficiency of the acetylcholine receptor results from mutations in genes coding any of the subunits of the acetylcholine receptor, with those related to the $\mu$ subunit being the most severe 5,15 . The clinical picture is characterized by ptosis, refractory marked ophthalmoparesis and severe muscle weakness of the limbs. There is generally a partially responsive pattern to pyridostigmine, amifampridine and albuterol use in clinical practice ${ }^{5,15,17}$. A very good response to pyridostigmine virtually rules out end plate Ach deficiency ${ }^{38}$. Our cases responded well with AChE inhibitors with history of reappearance of symptoms on stoppage of medication without adding any immunosuppressive therapy, plasma exchange or thymectomy.
Rapsyn concentrates and anchors the acetylcholine receptor in the postsynaptic membrane and is needed for the development of the junctional folds ${ }^{48}$. Most patients with rapsyn deficiency present in the first year of life, with a few presenting in childhood or adult life ${ }^{49}$. Both of our cases presented at $2^{\text {nd }}$ year of age. Rapsyn mutations produce a CMS in which respiratory distress, hypotonia, and poor feeding are usually present at birth. There is generalized weakness and ptosis but ophthalmoplegia is uncommon. Patients have a high-arched palate and may have arthrogryposis ${ }^{43}$. Infections can precipitate exacerbation of clinical manifestations ${ }^{50}$. In few patients prominent hyperlordosis can occur ${ }^{51}$. In our cases, there was hypotonia (more in case 1), generalized weakness and ptosis (both case), higharched palate \& hyperlordrosis (case 1) and history of exacerbation of symptoms during RTI.

\section{Conclusion}

Among the postsynaptic CMS, Rapsyn deficiency is most common and widely studied. Without genetic analysis it is not possible to differentiate among the different varieties of post synaptic CMS precisely but considering the all clinical and investigations scenario both of our cases mostly fits with the autosomal recessive, post synaptic CMS associated with Rapsyn deficiency.

\section{References}

1. Finsterer, J. Congenital myasthenic syndromes. Orphanet J Rare Dis 14, 57 (2019). https://doi.org/10.1186/s 13023-0191025-5

2. Souza PV, Batistella GN, Lino VC, Pinto WB, Annes M, Oliveira AS, Clinical and genetic basis of congenital myasthenic syndromes. Arq Neuropsiquiatr. 2016 Sep;74(9):750-760. doi: 10.1590/0004-282 X 20160106 .

3. Congenital Myasthenic Syndromes [internet]. Available from: https://rarediseases.org/rarediseases /congenital-myasthenic-syndromes/

4. Abicht A,Müller JS, Lochmüller H. Congenital Myasthenic Syndromes. GeneReviews, July 2016, https://www.ncbi.nlm.nih.gov/books/ NBK1168. 
5. Rodríguez Cruz PM, Palace J, Beeson D. Inherited disorders of the neuromuscular junction: an update. J Neurol. 2014;261(11):2234-43. doi:10.1007/s00415014-7520-7

6. Abicht A, Müller J S, Lochmüller H. Congenital Myasthenic syndromes. 2003 may 9 [updated 2016 Jul 14]. In: Adam MP, Ardinger HH, Pagon RA, Wallace SE, Bean LJH, Mefford HC, Stephens K, Amemiya A, Ledbetter N, editors. GeneReviews ${ }^{\circledR}$ [internet]. Seattle (WA): University of Washington, Seattle; 1993-2017. Available from http:// www.ncbi.nlm.nih.gov/books/NBK1168/.

7. Parr JR, Andrew MJ, Finnis M, Beeson D, Vincent $A$, Jayawant $S$. How common is childhood myasthenia? The UK incidence and prevalence of autoimmune and congenital myasthenia. Arch Dis Child. 2014;99(6):53942. doi:10.1136/archdischild-2013-304788

8. Engel AG. Congenital myasthenic syndromes. In: Katirji B, Kaminski HJ, Ruff RL, editors. Neuromuscular disorders in clinical practice. New York: Springer; 2014. p. 1073-90.

9. Lorenzoni PJ, Scola RH, Kay CS, Werneck LC. Congenital myasthenic syndrome: a brief review. Pediatr Neurol. 2012;46(3):141-8. doi:10. 1016/j.pediatrneurol.2011.12.001

10. Chang T, Cossins J, Beeson D. A rare c.183_187dupCTCAC mutation of the acetylcholine receptor CHRNE gene in a South Asian female with congenital myasthenic syndrome: a case report. BMC Neurol. 2016;16: 195.

11. Mihaylova V, Scola RH, Gervini B, Lorenzoni PJ, Kay CK, Werneck LC et al. Molecular characterisation of congenital myasthenic syndromes in Southern Brazil. J Neurol Neurosurg Psychiatry. 2010;81(9):973-7. doi:10.1136/jnnp.2009.177816

12. Harper CM. Congenital myasthenic syndromes. Semin Neurol. 2004;24: 111-23.

13. Abath Neto O, Heise CO, Moreno CA, Estephan EP, Mesrob L, Lechner D, Boland A, Deleuze JF, Oliveira AS, Reed UC,
Biancalana V, Laporte J, Zanoteli E. Nonlethal CHRNA1-related congenital Myasthenic syndrome with a homozygous null mutation. Can J Neurol Sci. 2017;44:125-7.

14. Berrih-Aknin S, Le Panse R. Myasthenia gravis: a comprehensive review of immune dysregulation and etiological mechanisms. J Autoimmun. 2014;52:90-100. doi:10.1016/ j.jaut. 2013.12.011

15. Engel AG, Shen XM, Selcen D, Sine SM. Congenital myasthenic syndromes: pathogenesis, diagnosis, and treatment. Lancet Neurol. 2015; 14(4):420-34. doi:10.1016/S1474-4422(14)70201-7

16. Geer $M$, Schotland $M$ : Myasthenia gravis in the new born. Paediatrics 1960; $26: 101$.

17. McQuillen MP: Familial limb girdle myasthenia. Brain 1966; 89 : 121-132.

18. Conomy JP, Levisohn M, Fanaroff A : Familial infantile myasthenia gravis : Cause of sudden death in young children. J Paediatrics 1975; $87: 428-430$.

19. Engel AG, Lambert EH, Gomez MR : A new myasthenic syndrome with end plate acetylcholinesterase deficiency, small nerve terminals and reduced acetylcholine release. Ann Neurol1971; 1 : 315-326.

20. Engel AG, Lambert EH, Mulder DM et al : A newly recognized congenital myasthenic syndrome attributed to a prolonged open time of the acetyl choline induced ion channel. Ann Neurol 1982; 11 : 553-569.

21. Engel AG, Walls TJ, Nagel $A$ et at : Newly recognized congenital myasthenic syndromes. I. Congenital paucity of synaptic vesicles and reduced quantal release II. High conductance fast channel syndrome. III. Abnormal acetylcholine receptor $(\mathrm{AChR})$ interaction with acetylcholine. IV. AChR deficiency and short channel open time. Progress in Brain Research1990; 84 : 125-137.

22. Engel AG, Hutchinson DO, Nakano $S$ et al : Myasthenic syndromes attributed to mutations affecting the epsilon sub unit of the 
acetylcholine receptor. Ann New York Academy of Science1993; 681 : 496-508.

23. Robertson WC, Chun RWM, Kornguth SE : Familial infantile myasthenia. Arch Neurol 1980; $37: 117-119$.

24. Morgan-Hughes JA, Lecky BRF, Landon DN et al : Alterations in the number and affinity of junctional acetylcholine receptors in a myopathy with tubular aggregates. A newly recognised receptor defect. Brain1991; 104 : 279-295.

25. Gieron MA, Korthals JK : Familial infantile myasthenia gravis : Report of 3 cases with follow up into adult life. Arch Neurol 1985; 42 : 143-144.

26. Mora M, Lambert EH, Engel AG : Synaptic vesicle abnormality in familial infantile myasthenia. Neurology1987; 37: 206-214.

27. Oosterhuis HJGH, Newsom-Davis J, Wokke $\mathrm{JHT}$ et al : The slow channel syndrome. Two New cases. Brain 1987; 110 : 1061-1079.

28. Beeson D, Palace J, Vincert A : Congenital myasthenic syndromes. Current Opinion in Neurology 1997;10: 402407.

29. Engel AG, Lambert EH : Congenital myasthenic syndromes. Electro Clin Neurophy1987; 39 : 91-102.

30. Sine SM, Ohno K, Bouzat C et al : Mutation of the acetylcholine receptor alpha sub unit causes a slow channel myasthenic syndrome by enhancing agonist binding affinity. Neuron1995; 15 : 229-239.

31. Camp S, Bon S, Li Y et al : Patients with congenital myasthenia associated with end plate acetylcholinesterase deficiency show normal sequence, mRNA splicing and assembly of catalytic sub units. J Clin Investigation 1995; 95 : 333-340.

32. Ohno K, Wang HL, Milone M et al : Congenital myasthenic syndrome caused by decreased agonist binding affinity due to mutation in the acetylcholine receptor epsilon sub unit. Neuron1996; 17 : 157-170.
33. Engle AG, Ohno K, Milone $M$ et al : New mutations in acetylcholine receptor sub unit genes reveal heterogeneity in the slow channel congenital myasthenic syndrome. Human Molecular Genetics1996; 5 : 12171227.

34. Engel AG, Ohno K, Bouzat $\mathrm{C}$ et al : End-plate acetylcholine receptor deficiency due to nonsense mutations in the epsilon sub unit. Ann Neurol 1996; 40 : 810-817.

35. Deconinck AE, Potter AC, Tinsley JM et al : Post synaptic abnormalities at the neuromuscular junctions of utrophin deficient mice. J Cell Biology 1997; 136 : 883-894.

36. Engel $A G$ : Myasthenic syndromes. In : Myology Basic and Clinical. Eds Engel AG, Franzini Armstrong C McGraw-Hill Inc, New York, 1994; 1798-1835.

37. Andrews PI, Massey JM, Sanders DB : Acetyl choline receptor antibodies in juvenile myasthenia gravis. Neurology 1993; 43 : 977-982.

38. Khwaja GA, Chowdhury D, Gupta M : Congenital myasthenic syndrome: report of four cases and brief review of literature. Neurol India 2000; 48 : 266-271.

39. Finnis, Maria F, and Sandeep Jayawant. "Juvenile myasthenia gravis: a paediatric perspective. "Autoimmune diseases vol. 2011 (2011): 404101. doi:10.4061/ 2011/ 404101

40. G. O. Skeie, S. Apostolski, A. Evoli et al., "Guidelines for treatment of autoimmune neuromuscular transmission disorders," European Journal of Neurology, vol. 17, no. 7, pp. 893-902, 2010.

41. Schneider CG,Gajdos P, Toyka PKV, Hohlfeld RR. "Corticosteroids for myasthenia gravis," Cochrane Database of Systematic Reviews, vol. 18, no. 2, p. CD002828, 2005.

42. Gronseth GS, Barohn RJ., "Practice parameter: thymectomy for autoimmune myasthenia gravis (an evidence-based review): report of the Quality Standards 
Subcommittee of the American Academy of Neurology," Neurology, vol. 55, no. 1, pp. 715, 2000.

43. Daroff RB, Bradley WG. (2012). Bradley's neurology in clinical practice. Philadelphia, PA, Elsevier/Saunders. P1909

44. Cossins J, Burke G, Maxwell S, Spearman $\mathrm{H}$, Man $\mathrm{S}$, et all. Diverse molecular mechanisms involved in AChR deficiency due to rapsyn mutations. Brain, Volume 129, Issue 10, October 2006, Pages 2773-2783, https:/ /doi.org/10.1093/brain/awl219

45. Ropper AH, Adams RD, Victor M, Brown RH (2019). Adams and Victor's principles of neurology. New York, McGraw-Hill Medical Pub. Division. P1484

46. Engel AG. Congenital Myasthenic syndromes in 2018. Curr Neurol Neurosci Rep. 2018;18(8):46. https://doi.org/10.1007/ s11910-018-0852-4.

47. Ohno K, Quiram P, Milone M, Wang HL, Harper MC, Pruitt JN 2nd et al. Congenital myasthenic syndromes due to heteroallelic nonsense/ missense mutations in the acetylcholine receptor $\mu$ subunit gene: identification and functional characterization of six new mutations. Hum Mol Genet. 1997;6(5):753-66. doi:10.1093/hmg/6.5.753

48. Ohno K, Engel AG, Shen X-M, et al. Rapsyn mutations in humans cause endplate acetylcholine receptor defi ciency and myasthenic syndrome. Am J Hum Genet 2002; 70: 875-88

49. Burke G, Cossins J, Maxwell S, et al. Rapsyn mutations in hereditary myasthenia. Distinct early- and late-onset phenotypes. Neurology 2003; 61: 826-828

50. Natera DD, Bestué M, Vilchez JJ, Evangelista T, Töpf A, García-Ribes A, Tiebas TMJ, García-Hoyos M, Ortez C, Camacho A, Jiménez $E$, Dusl M, Abicht A, Lochmüller $H$, Colomer J, Nascimento A. Long-term followup in patients with congenital myasthenic syndrome due to RAPSN mutations. Neuromuscul Disord. 2016;26:153-9.

51. Pavone $P$, Praticò AD, Pavone V, Falsaperla R. Congenital familial myasthenic syndromes: disease and course in an affected dizygotic twin pair. BMJ Case Rep 2013;2013. pii: bcr2012007651. doi: https://doi.org/ 10.1136/ bcr-2012-007651. 